\title{
Programa Mais Médicos - Esta é a solução?
}

\author{
Alexandre Marinho \\ Simone de Souza Cardoso \\ Vivian Vicente de Almeida
}

ABSTRACT | This text seeks to characterize the Brazilian public health program Mais Médicos, referring to the structure of primary health services in Brazil. The program's goal is to offer health services with a focus on improving the health of the population, via hiring more physicians. A preliminary econometric exercise that tries to explain a synthetic health indicator - infant mortality rate - through variables related to the provision of basic health services, as well as on the welfare of the population, was also conducted. The results show that hiring more physicians maybe a necessary - but not sufficient - condition to mitigate the serious public health problems that occur in Brazil.

KEY WORDS | Keywords: Program Mais Médicos, Infant mortality rate, hierarchical linear model.

RESUMO | O programa Mais Médicos, cujo objetivo é aumentar o acesso e a qualidade de serviços públicos básicos de saúde no Brasil com a contratação de mais médicos, foi o objeto de análise deste estudo. Também realizamos um exercício econométrico preliminar procurando explicar um indicador sintético de saúde - a taxa mortalidade infantil - por meio de variáveis relativas à provisão de serviços básicos de saúde e de indicadores das condições de vida da população. Avalia-se que elevar a oferta de profissionais de saúde pode ser uma condição necessária, mas não suficiente, para mitigar as complexas questões sanitárias que recaem sobre a população brasileira.

PALAVRAS-CHAVE | Programa Mais Médicos, taxa de mortalidade infantil, modelo hierárquico linear. 



\section{INTRODUÇ̃̃O}

A questão da má distribuição de médicos por territórios nacionais é amplamente discutida e debatida na literatura especializada. Trata-se, mesmo, de problema universal (Massmed, 2010, Japan-World Bank, 2013). No Brasil, Lacerda et. al. (2012) destacam que, enquanto 20\% da população brasileira habitam as capitais, $60 \%$ dos médicos trabalham nelas. O Conselho Federal de Medicina, em sua publicação sobre a Demografia Médica no Brasil (2013), alerta para o fato de que, apesar do crescimento exponencial dos profissionais de medicina nos últimos anos - levando, inclusive, o Brasil a ocupar taxas de médicos por mil habitantes comparáveis a países desenvolvidos - a questão da distribuição pelo território brasileiro ainda é um problema. Só para ilustrar, as regiões Norte (1,01 médicos por mil moradores (mpm)) e Nordeste (1,23 $\mathrm{mpm})$ apresentam indicadores bem distantes das regiões Sul (2,09 mpm), Centro-Oeste (2,05 mpm) e, especialmente, Sudeste (2,67 mpm) (CFM, 2013). Maciel Filho (2007) relata as principais iniciativas governamentais para melhora na distribuição de médicos já ocorridas no Brasil, com as razões para o insucesso ou sucesso de cada uma delas, com destaque positivo para o Projeto Rondon. Póvoa e Andrade (2006) apresentaram um modelo econométrico para explicar a localização dos médicos, no qual enfatizam a diversidade de determinantes, como a situação do cônjuge e o PIB local, para tal distribuição, e assinalam que a concentração de programas de residência médica nas regiões Sul e Sudeste do Brasil são decisivas para a preferência dos médicos em trabalhar nessas regiões. Neri e Soares (2002) destacam que a falta de acesso dos habitantes das cidades do interior, bem como a própria distância dessas localidades às capitais, onde comumente se concentram grande parte dos profissionais de medicina, configuram uma desigualdade relevante, que muitas vezes se traduz como um reflexo da desigualdade de renda. Esses autores chamam atenção, ainda, para o fato de que o acesso - ou falta dele - a serviços como saúde e educação pode impactar a renda permanente dos indivíduos, na medida em que afetam o capital produtivo. Adicionalmente, a distribuição das especialidades também é muito 
concentrada. Se, por um lado, pediatras, ginecologistas e obstetras respondem por, aproximadamente, 25\% das especialidades, o médico da família responde por 1,29\% (Lacerda et. al. op. cit., 2012). Esse resultado se revela particularmente relevante no contexto da análise do programa Mais Médicos. Parte da motivação do programa reside, justamente, no incentivo, tanto à interiorização da medicina, quanto da prática da medicina da família. E, a despeito da existência do Programa Estratégia de Saúde da Família (Programa de Saúde da Família - PSF) poucas foram as evidências encontradas na literatura de que esta é uma especialidade muito pouco incentivada, bem como pouco difundida pelo território brasileiro (Gonçalves et. al., 2009).

Dada essa má distribuição dos médicos no Brasil - tanto no que se refere ao quantitativo de profissionais nas regiões, quanto na especialização dos mesmos -, no dia 08 de Julho $2013^{1}$ o governo federal lançou o programa Mais Médicos. Fundamentando-se na necessidade de levar atendimento médico a cidades do interior, o programa tem por objetivo atrair médicos de várias nacionalidades, inclusive brasileiros, para atendimento nessas localidades. Inicialmente, o programa tinha por objetivo abrir cerca de 10 mil vagas para atuação exclusiva na área de atenção básica no Norte e Nordeste, periferias de grandes cidades e municípios do interior em todas as regiões do país. Mas a chegada de profissionais cubanos sob outras regras e regimes de contratação levantou uma série de questionamentos acerca do programa e suas reais motivações (Ministério da Saúde, 2013).

A implementação de programas e/ou políticas públicas que atraiam profissionais estrangeiros não é inédita. Também não é inédito o direcionamento deste tipo de política para a carreira de medicina. Países como o Canadá, Austrália, Inglaterra, dentre outros, já se utilizaram desse tipo de política pública para minorar ou eliminar a escassez de médicos e/ou a má distribuição desses profissionais pelo território nacional. Contudo, entre os pontos comuns deste tipo de programa nesses países está a oferta/garantia de pacotes de benefícios, não só para os médicos, mas para a família, incluindo emprego para os cônjuges, suporte escolar para os filhos, suporte psicológico e social, dentre outros. Não é inédita, também, a necessidade de ampliar a rede de proteção de saúde via contratação de médicos, estrangeiros ou não. Países como Estados Unidos e a própria Europa se deparam com iminentes déficits de médicos (Japan - World Bank (2013), Massmed (2010)).

Em meio a polêmicas, o programa tem sido alvo de críticas contundentes - principalmente por parte da classe médica - algumas defesas, e posições ainda indefinidas. Porém, ainda que permeiem debates ricos em críticas, uma análise mais detalhada se faz necessária. Este texto busca cumprir um duplo objetivo: (i) a avaliação da estrutura de atendimento na rede SUS; e (ii) inferir se a melhor política de saúde é a contratação de mais médicos. A meta deste texto, portanto, é tentar estimar, em que medida, o programa Mais Médicos seria, potencialmente, eficaz para melhorar a Atenção Básica no país, em especial, nos municípios mais pobres e/ou

1 O programa foi lançado pela Medida Provisória no ${ }^{\circ}$ 21, de 8 de julho de 2013, posteriormente convertida na Lei nº 12.871 , em 22 de Outubro de 2013. Fonte: http://www.planalto.gov.br/ccivil 03/ ato2011-2014/2013/Lei/L12871.htm 
interiorizados e, portanto, se esta seria a melhor solução. Para cumprir esta tarefa, o texto está dividido em cinco seções, além desta Introdução. Na segunda seção será realizada uma caracterização do referido programa, seguida de um mapa da estrutura da saúde no Brasil. Com isso, procura-se inferir, preliminarmente, o estado da saúde pública no Brasil e, ainda, as necessidades de cada estado e região no que tange a este setor. Em seguida, foi elaborado um exercício empírico simplificado, até por razões de concisão, procurando explicar um indicador sintético de saúde, a taxa de mortalidade infantil, por meio de variáveis relativas à provisão de serviços básicos de saúde, bem como relativos ao bem estar da população. E, por fim, são apresentadas as Considerações Finais.

\section{CARACTERIZACÃO DO PROGRAMA}

A análise dos possíveis impactos do programa e a própria distribuição do serviço de saúde no território brasileiro, demanda uma prévia e necessária, ainda que breve, caracterização do programa. Basicamente, o objetivo do programa é levar mais médicos ao interior e aos municípios mais pobres do Brasil visando melhorar a Atenção Básica à Saúde. As iniciativas do governo podem ser dividas em medidas direcionadas tanto para estudantes de medicina, quanto para médicos já formados.

No caso dos estudantes, inicialmente, o governo pretende criar, aproximadamente, 12 mil vagas em cursos de medicina até 2017. Na verdade, essa intenção já tinha sido evidenciada pelo governo federal desde o início da década de 2010 quando divulgou, no ano de 2012, um plano de ampliação de mais de 2.400 vagas em escolas médicas no Brasil até o ano de 2014 (Lacerda et. al., 2012). Além disso, pretende-se criar obrigatoriedade de dois anos de trabalho na rede pública - o Sistema Único de Saúde (SUS). Essa medida tinha, inicialmente, o objetivo de funcionar como um estágio, mas o programa foi modificado para funcionar como parte da residência. No caso de médicos já formados, o programa apresenta as características citadas no Quadro 1.

Quadro 1 | Programa Mais Médicos

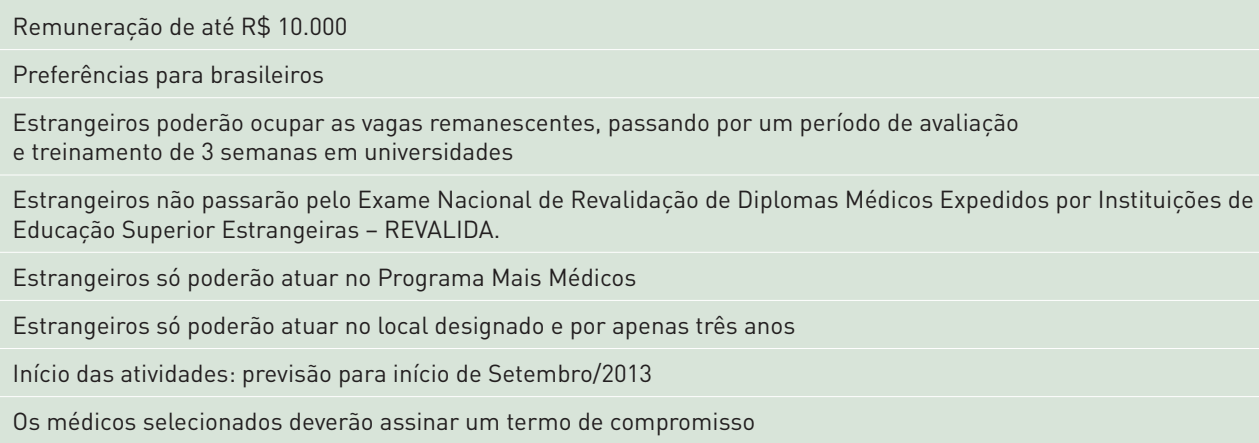

Fonte: Ministério da Saúde. Mais Médicos para o Brasil, Mais Saúde para Você, 2013. 


\subsection{Alguns números}

Nesta seção busca-se fazer um balanço sintético sobre a situação da atenção à saúde no Brasil, ao menos no que se refere à distribuição de recursos da saúde disponíveis à população. Para tal, entende-se por recursos de saúde variáveis relativas à infraestrutura disponível à população, no que se refere aos cuidados com a saúde: médicos, enfermeiros, hospitais, equipamentos, dentre outros.

O levantamento relativo à infraestrutura disponível em termos de serviços de saúde atende a um duplo objetivo. Num primeiro momento, a disponibilidade de mais médicos atuando no Sistema Único de Saúde, em principio, pode parecer aceitável e, até, desejável. Assim, torna-se necessário avaliar em que medida o país necessita de mais médicos. Outro ponto refere-se à distribuição dos médicos e da população no país, por Unidade da Federação. Isso porque a avaliação das necessidades de médicos no Brasil tem que refletir as especificidades de cada região. Primeiramente, será avaliado o número de médicos por mil habitantes do Brasil comparativamente a alguns países selecionados, conforme Tabela 1 .

Tabela 1 | Distribuição dos Médicos pelos Países Selecionados - 2013

\begin{tabular}{|l|l|}
\hline Médicos por $\mathbf{1 . 0 0 0}$ Habitantes & $\mathbf{1 , 8 0}$ \\
\hline Brasil & 3,20 \\
\hline Argentina & 3,70 \\
\hline Uruguai & 3,90 \\
\hline Portugal & 4,00 \\
\hline Espanha & 2,70 \\
\hline Reino Unido & 3,00 \\
\hline Austrália & 3,50 \\
\hline Itália & 3,60 \\
\hline Alemanha & 2013. \\
\hline
\end{tabular}

Fonte: Ministério da Saúde. Mais Médicos para o Brasil, Mais Saúde para Você, 2013.

A Tabela 1 mostra que, tanto quando comparadas com realidades econômicas similares - Argentina, por exemplo - quando como comparados com países desenvolvidos e, em certa medida, referências em termos de provisão do serviço de saúde em sistemas de saúde universais e gratuitos - Reino Unido - nosso resultado fica bem aquém do desejado. Só a leitura deste indicador poderia suscitar um parecer positivo à implementação do programa em análise, uma vez que, teoricamente, dispõe-se de um número muito menor de médicos por habitantes que qualquer um dos países citados. Contudo, uma análise mais detalhada se faz necessária por, ao menos, três razões. A primeira diz respeito à própria natureza e motivação de criação do programa. Este foi elaborado com o intuito de levar médicos para as regiões mais necessitadas. Assim, analisar apenas o indicador brasileiro agregado não revela as necessidades específicas 
de cada região. Segundo, pela própria natureza heterogênea do Brasil, onde coexistem cidades e estados muito densos com municípios distantes com poucos habitantes e, em alguns casos, sem médico algum. E, por fim, pelo fato de que o número de médicos por habitantes pode não ser suficiente para revelar a real situação da oferta de saúde em um determinado local. Uma elevada quantidade de médicos disponíveis pode não significar um bom acesso a serviços públicos de saúde. Portanto, para que a avaliação do estado da saúde pública no Brasil seja mais efetiva em termos de médicos por habitantes, é apresentada na Tabela 2, a distribuição de médicos por mil habitantes por Unidade da Federação.

Tabela 2 | Distribuição dos Médicos pelos Estados Brasileiros - 2013

\begin{tabular}{|l|l|l|l|}
\hline Médicos por $\mathbf{1 . 0 0 0}$ Habitantes & & & \\
\hline Acre & 1,08 & Paraíba & 1,38 \\
\hline Alagoas & 1,24 & Paraná & 1,87 \\
\hline Amapá & 0,95 & Pernambuco & 1,57 \\
\hline Amazonas & 1,12 & Piauí & 1,05 \\
\hline Bahia & 1,25 & Rio de Janeiro & 3,62 \\
\hline Ceará & 1,16 & Rio Grande do Norte & 1,43 \\
\hline Distrito Federal & 4,09 & Rio Grande do Sul & 2,37 \\
\hline Espírito Santo & 2,17 & Rondônia & 1,19 \\
\hline Goiás & 1,73 & Roraima & 1,38 \\
\hline Maranhão & 0,71 & Santa Catarina & 1,98 \\
\hline Mato Grosso & 1,26 & São Paulo & 2,64 \\
\hline Mato Grosso do Sul & 1,69 & Sergipe & 1,42 \\
\hline Minas Gerais & 2,04 & Tocantins & 1,36 \\
\hline Pará & 0,84 & Total & 1,80 \\
\hline
\end{tabular}

Fonte: Ministério da Saúde. Mais Médicos para o Brasil, Mais Médicos para Você, 2013.

A análise da Tabela 2 apresenta uma série de conclusões que merecem destaque. A primeira é a de que é necessário melhorar a distribuição dos médicos no Brasil. Ainda que os próprios estados possam apresentar desigualdades internas, a simples desagregação entre unidades da federação já evidencia uma desigualdade muito grande na distribuição de médicos por habitantes entre os estados brasileiros. Se, por exemplo, utiliza-se a média brasileira (1,80 mpm) como referência, apenas quatro unidades da federação - Paraná, Rio de Janeiro, Santa Catarina e São Paulo - além do Distrito Federal, é que ultrapassam este indicador.

Observando a Figura 1, percebe-se que mais de 40\% da população brasileira tem um acesso potencial limitado à cobertura médica, com menos de um médico disponível para cada mil habitantes. 
Figura 1 | Distribuição do percentual da população segundo a cobertura médica/1000 hab. no Brasil em 2010

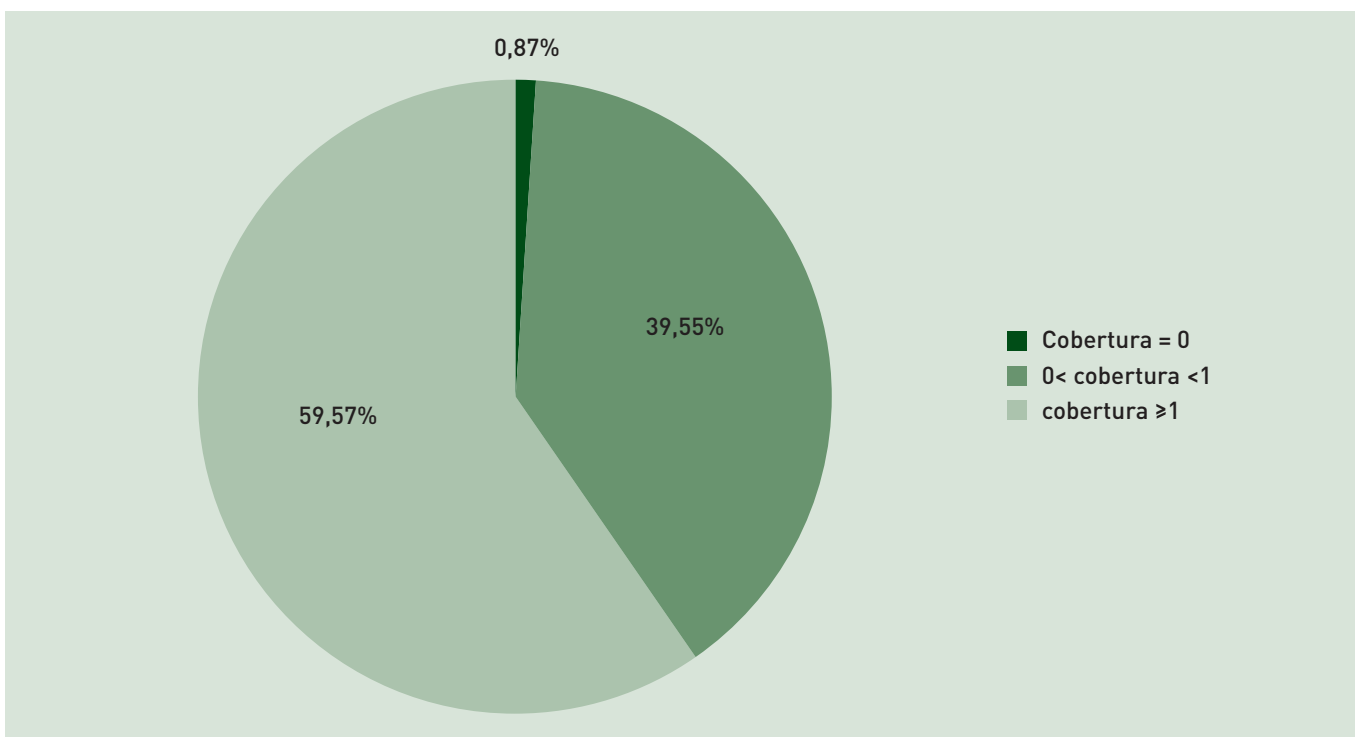

Fonte: Cadastro Nacional dos Estabelecimentos de Saúde do Brasil - CNES. Elaboração dos autores

Ainda que a falta de médicos constitua um problema per si e demande atenção e políticas voltadas para a mitigação deste problema - motivação do governo federal para a elaboração e implementação do programa em análise - muitos especialistas têm alertado para o fato de que a contratação de mais médicos não seria suficiente para melhorar a provisão dos serviços de saúde, caso não fosse acompanhada de uma melhora na infraestrutura do setor. Portanto, o próximo passo é avaliar o setor de saúde de um ponto de vista estrutural. Traçando, para tal, uma análise descritiva com base nos números relativos à infraestrutura do sistema de saúde brasileiro - público e privado. Para iniciar essa análise, destaca-se o Quadro 2, o qual descreve as variáveis relativas à estrutura do sistema de saúde.

Quadro 2 | Análise de variáveis dos municípios brasileiros relativas à infraestrutura do sistema de saúde - 2012

\begin{tabular}{|c|c|c|c|c|c|c|c|}
\hline Variáveis & Obs. & Média & Desvio Padrão & Min. & Max & \multicolumn{2}{|c|}{ Intervalo de confiança $95 \%$} \\
\hline $\begin{array}{l}\text { \% Programa Saúde da } \\
\text { Família (PSF) }\end{array}$ & 5565 & 83,415 & 43,64 & 0 & 393,52 & 82,268 & 84,562 \\
\hline Despesa per capita (R\$) & 5565 & 383,945 & 174,41 & 0 & 1980,19 & 379,362 & 388,529 \\
\hline $\begin{array}{l}\text { Equipamentos existentes } \\
\text { por mil habitantes }\end{array}$ & 5565 & 2,976 & 2,623 & 0 & 41,143 & 2,907 & 3,045 \\
\hline $\begin{array}{l}\text { Equipamento SUS } \\
\text { por mil habitantes }\end{array}$ & 5565 & 1,579 & 1,227 & 0 & 12,5 & 1,546 & 1,611 \\
\hline $\begin{array}{l}\text { Enfermeiro Superior } \\
\text { por mil habitantes }\end{array}$ & 5565 & 0,551 & 0,262 & 0 & 3,632 & 0,544 & 0,558 \\
\hline $\begin{array}{l}\text { Téc. Enfermagem } \\
\text { por mil habitantes }\end{array}$ & 5565 & 0,726 & 0,744 & 0 & 6,513 & 0,706 & 0,745 \\
\hline $\begin{array}{l}\text { Total de Leitos } \\
\text { por mil habitantes }\end{array}$ & 5565 & 1,854 & 2,178 & 0 & 31,289 & 1,797 & 1,912 \\
\hline
\end{tabular}

Fonte: Cadastro Nacional dos Estabelecimentos de Saúde do Brasil - CNES; Sistema de Informações sobre Orçamentos Públicos em Saúde - SIOPS; Sistema de Informação da Atenção Básica - SIAB. Elaboração dos autores. 
Observando os dados municipais do Quadro 2, o que se percebe é que há uma disparidade muito grande entre as cidades brasileiras, no que se refere à estrutura de saúde disponível à população. Por exemplo, em termos de leitos por mil habitantes, apesar de a média ser de aproximadamente duas unidades por mil habitantes, observa-se um máximo de trinta e uma unidades por mil habitantes. Esta mesma análise pode ser estendida para os equipamentos existentes. Esta variável, inclusive, é passível de uma análise mais detalhada. Nas Figuras 2, 3 e 4 evidenciase a evolução temporal do percentual de equipamentos disponíveis e em uso no Brasil e no SUS.

Figura 2 | Grupo de Equipamentos disponíveis no SUS por Ano/mês 2005 a 2013

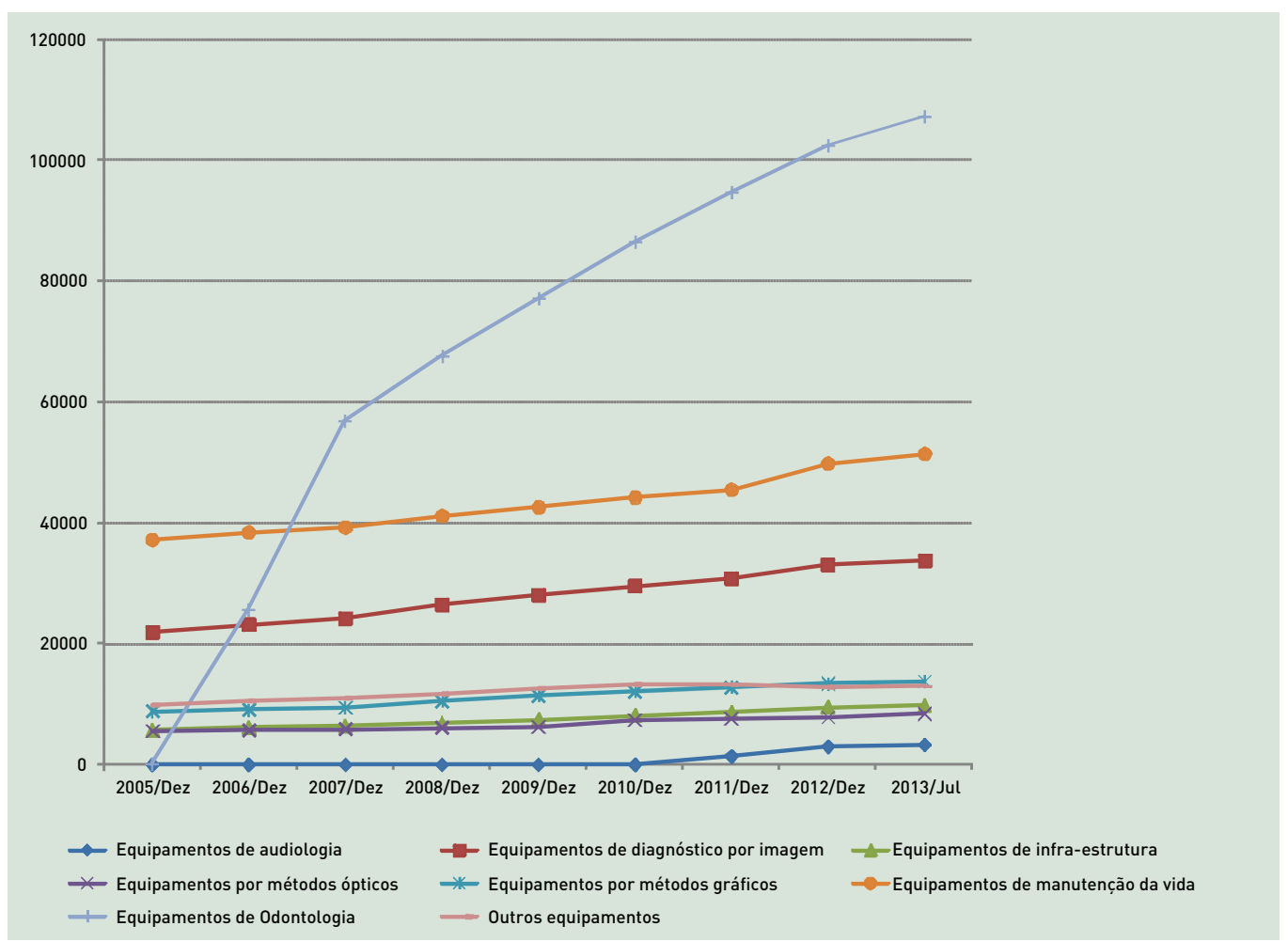

Fonte: Ministério da Saúde - Cadastro Nacional dos Estabelecimentos de Saúde do Brasil - CNES. Elaboração dos autores. 


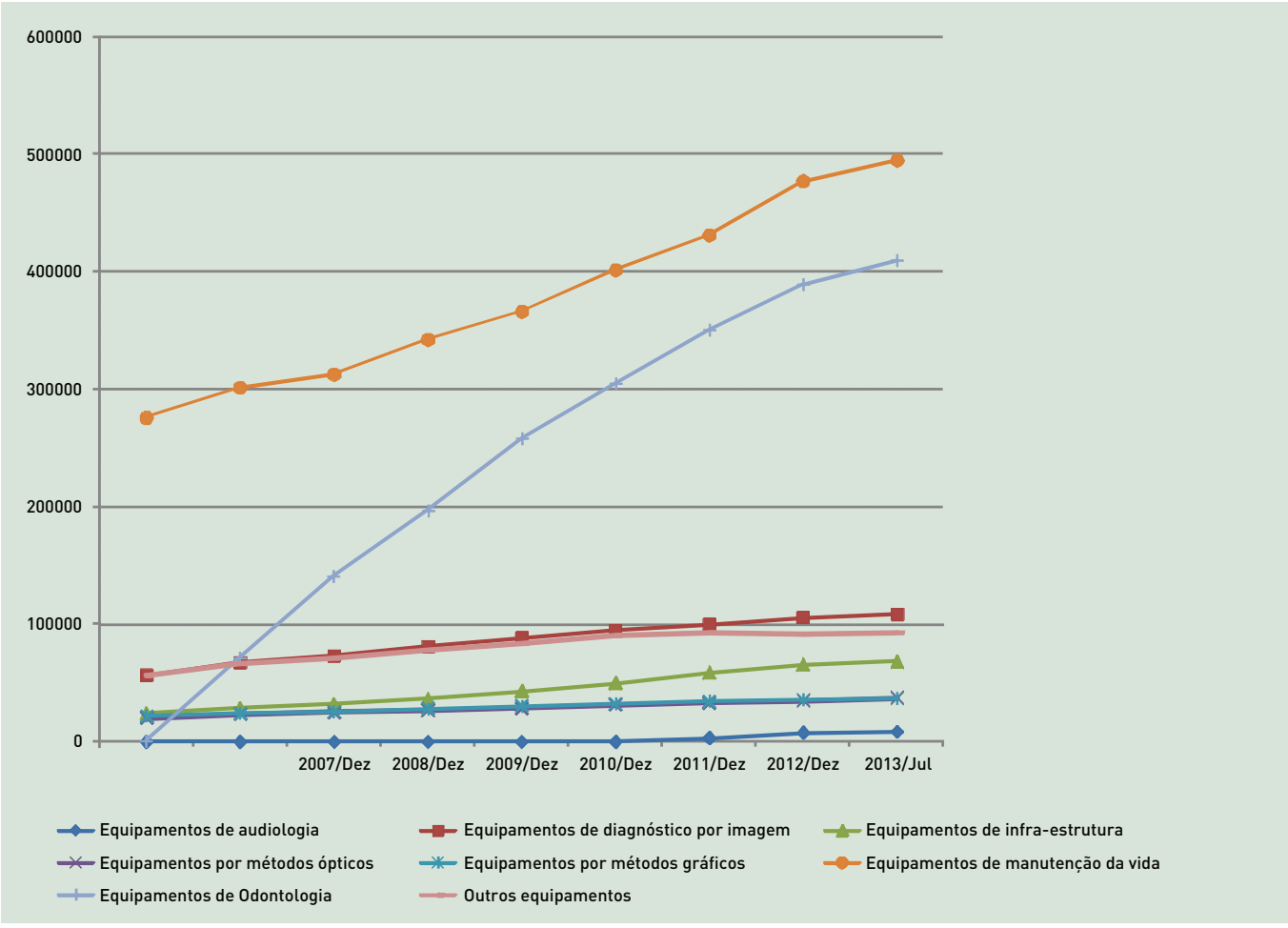

Fonte: Ministério da Saúde - Cadastro Nacional dos Estabelecimentos de Saúde do Brasil - CNES. Elaboração dos autores.
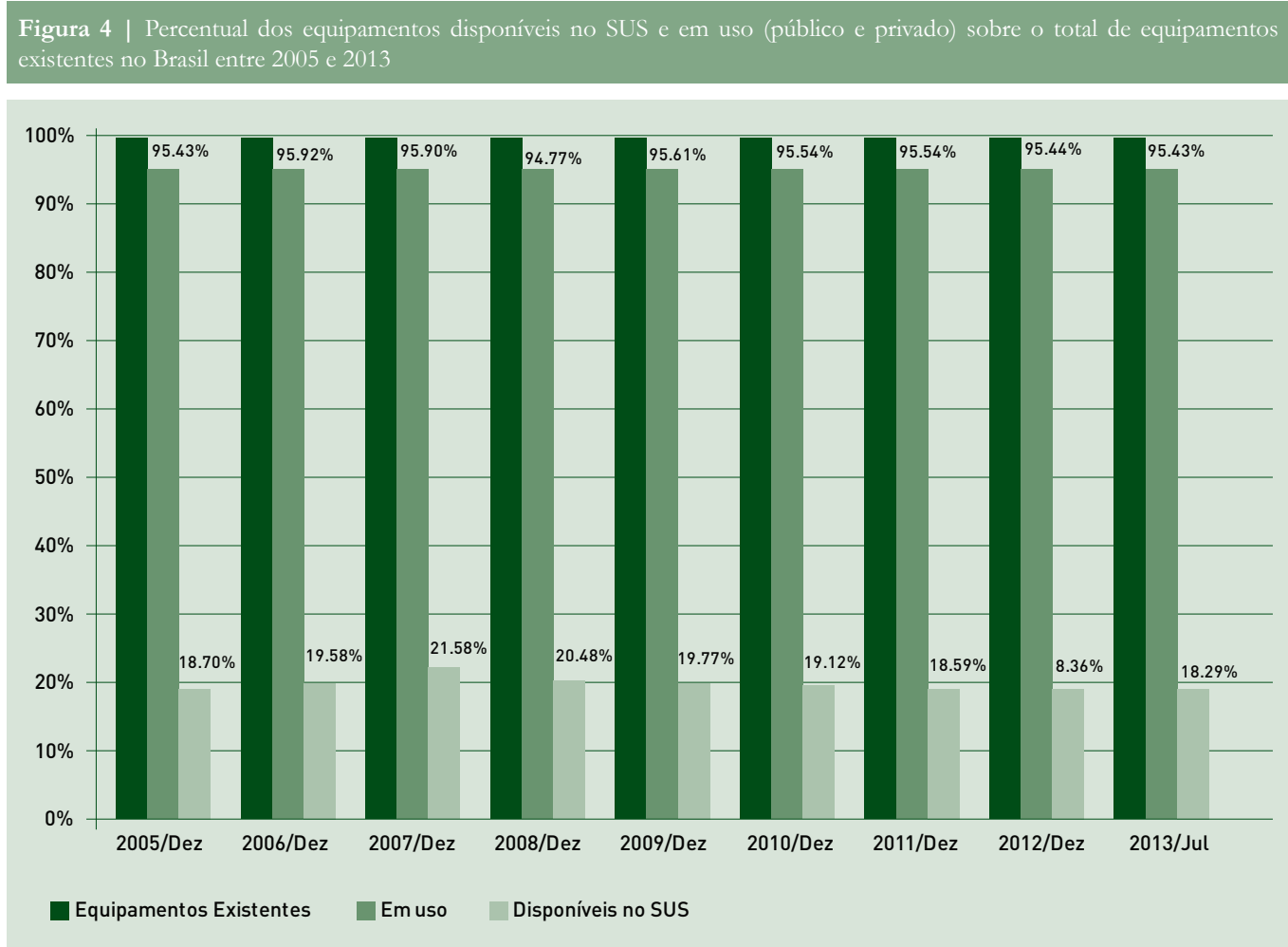

Fonte: Ministério da Saúde - Cadastro Nacional dos Estabelecimentos de Saúde do Brasil - CNES. Elaboração dos autores. 
Na Figura 2 e na Figura 3, como é possível perceber, a oferta de equipamentos no SUS é crescente entre os anos de 2005 e 2013. Na Figura 4 observa-se que, dos equipamentos existentes, a taxa de utilização é, em média, de 95\%. Porém, destes equipamentos disponíveis à população - públicos e privados - apenas 19\%, em média, estão disponíveis no SUS. Ou seja, a percepção de que há uma falta de infraestrutura física no sistema de saúde público brasileiro comprova-se acertada.

Em suma, o que é possível concluir, até este ponto, é que, de fato, há crônico problema na distribuição dos serviços de saúde no país. Essa má distribuição, que por vezes se reflete na desigualdade de renda, e por vezes se confunde com a mesma, tem impactos nocivos ao bem-estar da população, revelando uma necessidade de melhorias na provisão deste bem meritório. Portanto, o exercício de avaliar o programa Mais Médicos é ainda mais justificado quando refletido o retrato da desigual atenção à saúde no país. O próximo passo, então, é inferir se o problema da má distribuição, que acaba por se transformar na falta de acesso de parte da população à saúde, pode ser resolvido com apenas mais médicos.

\section{DESCRIC̣ÃO DOS MODELOS UTILIZADOS E OS RESULTADOS OBTIDOS.}

\subsection{As variáveis e os modelos utilizados}

Observando os dados, é possível perceber que a escassez de médicos é relativa. Enquanto, de fato, muitos estados e municípios carecem desta prestação de serviços, outros lugares apresentam indicadores inclusive maiores que países desenvolvidos, como no caso de Brasília. Mais grave que a escassez de médicos, contudo, parece ser a falta de estrutura em termos de equipamentos do SUS comparativamente à rede privada. Dos equipamentos em uso, mais de 80\% (oitenta por cento) são da rede privada. Mas, ainda que um diagnóstico descritivo seja bastante útil e informativo para o processo de tomada de decisão, respostas para a pergunta mais básica e determinante deste estudo - contratar mais médicos, ceteris paribus, é a melhor opção para melhorar a saúde da população brasileira? - não foi, ainda, respondida. Para tentar responder a este questionamento realizou-se alguns exercícios empíricos preliminares. O objetivo, aqui, foi tentar inferir, em que medida, a infraestrutura na prestação de serviços de saúde é, ou não, determinante para a saúde da população em um modelo de regressão em painel ${ }^{2}$, para os 5.565 municípios brasileiros, agrupados nas vinte e sete unidades da federação. Para isso, foi escolhido um indicador sintético e clássico na literatura de saúde, a Taxa de mortalidade infantil que, inclusive, reflete, em certa medida, as condições da Atenção Básica à Saúde no Brasil e em outros países (Afonso e St. Aubyn (2005). O exercício, portanto, foi tentar explicar essa variável utilizando as seguintes variáveis explicativas:

2 O modelo econométrico utilizado foi o modelo hierárquico em dois níveis: unidades da federação e municípios, sendo o estado o nível mais agregado. 


\begin{tabular}{l|l}
\hline Expectativa de anos de estudo & Médicos per capita \\
\hline Taxa de analfabetismo & Enfermeiros com nível superior \\
\hline Despesa em saúde per capita & Técnico de enfermagem per capita \\
\hline Equipamentos existentes per capita & Leitos per capita \\
\hline Equipamentos SUS per capita & \% Água e esgoto adequado \\
\hline Percentual de cobertura do PSF & Renda per capita média \\
\hline
\end{tabular}

As variáveis utilizadas foram retiradas das fontes de dados descritas a seguir. A informação sobre a população residente foi retirada do IBGE - Censos Demográficos/ DATASUS. O Percentual de cobertura do PSF (Proporção da população cadastrada pela Estratégia Saúde da Família) teve como fonte o Sistema de Informação da Atenção Básica - SIAB. A Despesa total com saúde per capita foi retirada do SIOPS - Sistema de Informações sobre Orçamentos Públicos em Saúde. Já para a Taxa de Mortalidade Infantil foi usado o número de óbitos infantis por residência sobre os nascidos vivos por residência da mãe. Os dados foram extraídos do MS/SVS/DASIS - Sistema de Informações sobre Mortalidade - SIM e do Sistema de Informações sobre Nascidos Vivos - SINASC. O método de cálculo utilizado para esta variável foi o seguinte:

\section{$T M I=\frac{\text { Número de óbitos de residentes com menos de um ano de idade }}{\text { Número de nascidos vivos de mães residentes }} * 1000$}

As outras variáveis utilizadas apresentam a seguinte construção.

Índice de Desenvolvimento Humano Municipal: Média geométrica dos índices das dimensões Renda, Educação e Longevidade, com pesos iguais.

Renda per capita média: Razão entre o somatório da renda de todos os indivíduos residentes em domicílios particulares permanentes e o número total desses indivíduos. Valores em reais de 01/agosto de 2010.

\% Água e esgoto adequados (Percentual de pessoas em domicílios com abastecimento de água e esgotamento sanitário adequados): $100 \%$ - Razão entre as pessoas que vivem em domicílios cujo abastecimento de água não provê de rede geral e cujo esgotamento sanitário não é realizado por rede coletora de esgoto ou fossa séptica e a população total residente em domicílios particulares permanentes multiplicados por $100 .^{3}$

Para calcular o percentual das pessoas em domicilio com abastecimento de água e esgotamento sanitário adequado foi considerado o complementar:

100\% - percentual de pessoas em domicilios com abastecimento de água e esgotamento sanitário inadequado

3 São considerados apenas os domicílios particulares permanentes. 
Os quatro itens listados anteriormente foram extraídos do site do Programa das Nações Unidas para o Desenvolvimento (PNUD), Atlas 2013.

Os indicadores: Médicos por mil habitantes (atende ou não no SUS); Equipamentos Existentes e Equipamentos disponiveis no SUS ambos por 1.000 habitantes; Leitos per capita (leitos de internação e UTI por mil habitantes), Enfermeiros com ocupação de nivel superior por 1.000 habitantes (atende ou não no SUS); e Técnico de Enfermagem por mil habitantes (atende ou não no SUS) foram retirados do Cadastro Nacional dos Estabelecimentos de Saúde do Brasil - CNES. Essas variáveis foram calculadas da seguinte forma:

Número de profissionais, da categoria de saúde específica ou equipamentos

População total residente * 1000

\subsection{Discussão dos resultados}

Nas tabelas 3 e 4 a seguir, são expostos os resultados dos modelos econométricos de regressão utilizados. Cada coeficiente explicita a correlação entre a Taxa de Mortalidade Infantil (TMI) as variáveis explicativas (determinantes). Variáveis com coeficientes positivos aumentam a TMI e vice-versa. Quanto maior a magnitude do coeficiente maior será o impacto da variável sobre a TMI e vice-versa. Serão consideradas relevantes as variáveis estatisticamente significativas ao nível de $5 \%$ (valor de prova $\mathrm{P} \leq 0,05$ ).

Quando avaliados os determinantes da Taxa de Mortalidade Infantil vê-se que as variáveis significativas são aquelas relativas à infraestrutura básica, bem como o nível educacional da população. Esse resultado é coerente e consistente, tanto com as expectativas deste estudo, quanto com a literatura (Barros et. al., 2010). Ceteris paribus, a sobrevivência de uma criança está muito mais relacionada ao ambiente em que nasce, bem como a provisão de cuidados básicos, do que com um aparato médico sofisticado e abundante. Esta informação é reforçada quando observada a Tabela 3, mostrada a seguir, em que as variáveis significativas para explicar a taxa de mortalidade infantil foram, no modelo completo (modelo cheio) as variáveis: Expectativas de Anos de Estudo e Técnicos de Enfermagem per Capita.

Esta análise é reforçada quando observado o modelo reduzido obtido pelo critério de informação de Akaike inclusivo - AIC. O AIC é uma estimativa da logverossimilhança negativa dos modelos, ponderada para o número de parâmetros estimados. Para mais detalhes sobre o método AIC, ver Maddala (2001), especialmente o capítulo $12^{4}$ e Wang e Jamison (2005). 4 O AIC é uma estimativa da logverossimilhança negativa dos modelos, ponderada para o número de parâmetros estimados. Consequentemen-
te, o modelo com menor valor de AIC é o mais apropriado sendo que: $\mathrm{AIC}=\square 2 \log (\mathrm{L}) / \mathrm{n}+(2 \mathrm{~K} / \mathrm{n})(5)$ onde: $\mathrm{L}=$ máxima verossimilhança; 
Tabela 3 | Modelo com variáveis explicativas inseridas - modelo cheio | Número de observações: 5565 | Número de grupos: 27

\begin{tabular}{|c|c|c|c|c|c|c|c|}
\hline $\begin{array}{l}\text { Taxa de Mortalidade } \\
\text { Infantil }\end{array}$ & Coeficientes & Erro-Padrão & DF & $T$ & $P>|z|$ & \multicolumn{2}{|c|}{ Intervalo de confiança $95 \%$} \\
\hline Intercepto & 20,406 & 2,889 & 5526 & 7,063 & 0,000 & 14,742 & 26,069 \\
\hline$\%$ de cobertura do PSF & $-0,008$ & 0,005 & 5526 & $-1,646$ & 0,100 & $-0,017$ & 0,002 \\
\hline Despesa per capita & $-0,002$ & 0,001 & 5526 & $-1,445$ & 0,148 & $-0,005$ & 0,001 \\
\hline Renda per capita média & $-0,003$ & 0,002 & 5526 & $-1,829$ & 0,068 & $-0,006$ & 0,000 \\
\hline $\begin{array}{l}\text { Expectativa de anos de } \\
\text { estudo }\end{array}$ & $-0,628$ & 0,231 & 5526 & $-2,716$ & 0,007 & $-1,081$ & $-0,175$ \\
\hline $\begin{array}{l}\text { Taxa de analfabetismo - } \\
18 \text { anos ou mais }\end{array}$ & 0,068 & 0,036 & 5526 & 1,883 & 0,060 & $-0,003$ & 0,138 \\
\hline $\begin{array}{l}\text { Equipamentos existentes } \\
\text { per capita }\end{array}$ & $-0,148$ & 0,131 & 5526 & $-1,137$ & 0,256 & $-0,404$ & 0,108 \\
\hline $\begin{array}{l}\text { Equipamento SUS } \\
\text { per capita }\end{array}$ & 0,325 & 0,234 & 5526 & 1,387 & 0,166 & $-0,134$ & 0,784 \\
\hline Médicos per capita & $-0,016$ & 0,448 & 5526 & $-0,037$ & 0,971 & $-0,894$ & 0,861 \\
\hline $\begin{array}{l}\text { Enfermeiro superior } \\
\text { per capita }\end{array}$ & 0,658 & 0,887 & 5526 & 0,742 & 0,458 & $-1,081$ & 2,397 \\
\hline $\begin{array}{l}\text { Técnicos de Enfermagem } \\
\text { per capita }\end{array}$ & 0,769 & 0,310 & 5526 & 2,484 & 0,013 & 0,162 & 1,376 \\
\hline Total de Leitos per capita & $-0,042$ & 0,101 & 5526 & $-0,413$ & 0,679 & $-0,240$ & 0,156 \\
\hline $\begin{array}{l}\text { \% Água e esgoto } \\
\text { adequados }\end{array}$ & 0,009 & 0,021 & 5526 & 0,410 & 0,682 & $-0,033$ & 0,050 \\
\hline \multicolumn{8}{|l|}{ Efeitos aleatórios } \\
\hline Parâmetros & Estimativas & & & & & \multicolumn{2}{|c|}{ Intervalo de confiança $95 \%$} \\
\hline DP (intercepto) & 1,045 & & & & & 0,765 & 1,426 \\
\hline DP (Resíduos) & 14,113 & & & & & 13,853 & 14,379 \\
\hline Correlação intraclasse & 0,005 & & & & & & \\
\hline AIC & 45340,550 & & & & & & \\
\hline
\end{tabular}

Fonte: elaboração dos autores.

A relação entre um maior nível educacional e melhores condições de saúde, aqui analisadas pela (redução da) Taxa de Mortalidade Infantil, não é inédita. A redução do analfabetismo e o aumento da escolaridade têm relação direta com a redução da mortalidade como observado no modelo cheio e ratificado pela significância das variáveis, bem como os valores e os sinais dos coeficientes de ambos indicadores. Justamente por se tratar de um indicador sintético, a taxa de mortalidade apresenta relação com as variáveis estruturais e com a educação, e as condições de vida. Essas afirmações são ratificadas, em grande medida, pela significância das referidas variáveis no modelo cheio, bem como pelos sinais associados aos respectivos coeficientes. Como é possível perceber, as variáveis Taxa de Analfabetismo - 18 anos ou mais (com sinal positivo); e Expectativa de Anos de Estudo (com sinal negativo) apresentam sinais convergentes à expectativa teórica de redução da TMI bem como a Cobertura do Programa de Saúde da Família - PSF, também com sinal negativo. Como será visto adiante, a única variável que diverge, em termos do sinal do coeficiente inicialmente esperado é a proporção dos Técnicos de Enfermagem per capita. Essa aparente dissonância será mais bem explicada a seguir.

$\mathrm{K}=$ número de parâmetros do modelo; $\mathrm{n}=$ número de observações. Quando o ajuste é bom o modelo explica boa parte da variação total e, consequentemente, o valor do coeficiente de determinação $\mathrm{R}^{2}$ é próximo da unidade. Foi utilizado o software livre $\mathrm{R}$. 
Tabela 4 | Modelo com variáveis explicativas inseridas - modelo reduzido | Número de observações: 5565 | Número de grupos: 27

\begin{tabular}{|c|c|c|c|c|c|c|c|}
\hline $\begin{array}{l}\text { Taxa de Mortalidade } \\
\text { Infantil }\end{array}$ & Coeficientes & Erro-Padrão & DF & $\mathbf{T}$ & $P>|z|$ & \multicolumn{2}{|c|}{ Intervalo de confiança 95\% } \\
\hline Intercepto & 18,346 & 2,364 & 5535 & 7,762 & 0,000 & 13,712 & 22,979 \\
\hline $\begin{array}{l}\text { Expectativa de anos de } \\
\text { estudo }\end{array}$ & $-0,683$ & 0,225 & 5535 & $-3,030$ & 0,003 & $-1,124$ & $-0,241$ \\
\hline $\begin{array}{l}\text { Taxa de analfabetismo - } \\
18 \text { anos ou mais }\end{array}$ & 0,120 & 0,027 & 5535 & 4,433 & 0,000 & 0,067 & 0,174 \\
\hline $\begin{array}{l}\text { Técnicos de Enfermagem } \\
\text { per capita, }\end{array}$ & 0,603 & 0,282 & 5535 & 2,140 & 0,032 & 0,051 & 1,155 \\
\hline \multicolumn{8}{|l|}{ Efeitos aleatórios } \\
\hline Parâmetros & Estimativas & & & & & \multicolumn{2}{|c|}{ Intervalo de confiança 95\% } \\
\hline DP (intercepto) & 1,241 & & & & & 0,717 & 2,146 \\
\hline DP (Resíduos) & 14,113 & & & & & 13,852 & 14,378 \\
\hline Correlação intraclasse & 0,008 & & & & & & \\
\hline AIC & 45292,250 & & & & & & \\
\hline
\end{tabular}

Fonte: elaboração dos autores.

O modelo reduzido (Tabela 4) contém apenas as variáveis significativas do modelo mais completo (modelo cheio). As variáveis dependentes utilizadas, neste caso, são: Expectativa de Anos de Estudo, e Técnicos de Enfermagem per capita e variável Taxa de analfabetismo - 18 anos on mais. Note-se que, em ambos os modelos, as variáveis Médicos per capita; Equipamentos do SUS per capita; e Equipamentos Existentes per capita não são significativos. É necessário destacar que a variável relativa ao número de técnicos de enfermagem apresenta um sinal contrário ao que poderia ser esperado, ou seja, apresenta uma relação negativa com a taxa de mortalidade em ambos os modelos. Esse fenômeno pode ser compreendido com a compreensão de que, nos municípios onde há maior mortalidade, em geral, se concentram, em maior escala, os profissionais de menor custo, desacompanhados dos demais elementos necessários ao bom funcionamento do sistema de saúde, pela tentativa malfadada de atender à população de forma precária. No Apêndice do presente texto é possível ver que existe uma correlação negativa entre Médicos per capita e Cobertura Percentual do PSF. Sabe-se que a cobertura do PSF é maior nos municípios e regiões de menor renda e desenvolvimento, onde os médicos são mais escassos. O PSF (ou Estratégia de Saúde da Família (ESF)) é uma das principais políticas públicas de prestação de cuidados básicos à saúde no Brasil. O que a correlação negativa indicaria seria um descasamento entre o PSF e a presença de médicos. Conforme já assinalado, o médico da família responde por apenas 1,29\% da força de trabalho médica no Brasil (Lacerda et. al. op. cit., 2012). Conjectura-se, assim, que tais fenômenos poderiam indicar que os problemas de saúde estão recaindo sobre profissionais não médicos (os quais, nosso estudo indica, seriam os Técnicos de Enfermagem) que, sem o apoio e sem a estrutura necessária, não estão conseguindo resolver a contento os problemas de saúde da população, mas estão sendo utilizados como substitutos inadequados para os demais insumos. Além disso, é possível perceber que as outras duas variáveis (Taxa de analfabetismo - 18 anos ou mais; e Expectativa de anos de estudo) que explicam a mortalidade infantil são aquelas relativas à educação da população. Tal achado ratificaria a 
suposição inicial de que o nível educacional da população é um importante determinante das condições gerais de saúde pública no Brasil, além da estrutura geral de prestação de serviços de saúde (os médicos incluídos).

\section{CONSIDERACุÕES FINAIS}

A justificativa para implementação do Programa Mais Médicos é que há, não só, uma desigualdade, mas uma escassez muito desigual, na distribuição de profissionais de medicina pelo território brasileiro. Essa justificativa encontra respaldo tanto na literatura, como em na análise dos dados relacionados com a presença de médicos no Brasil. Mas, ainda que este seja um problema relevante, o objetivo de promover o bem-estar e melhorar as condições de saúde da população não necessariamente seria sanado, apenas, com a provisão de mais médicos para a população e territórios carentes. Muitas das discussões sobre o programa destacam, principalmente, a escassez de infraestrutura do SUS o que, portanto, acabaria por tornar inócua a presença de mais profissionais. E, de fato, quando traçamos uma análise descritiva percebe-se que há, realmente, uma desigualdade entre os equipamentos disponíveis e aqueles pertencentes ao sistema de saúde supramencionado.

Os resultados econométricos, que são reconhecidos como preliminares, evidenciaram que, dentre os determinantes da Taxa de Mortalidade Infantil, as variáveis relativas à estrutura social, de modo específico as variáveis relativas à educação (Taxa de analfabetismo - 18 anos on mais; e Expectativa de Anos de Estudo) foram marcadamente significativas e são positivamente correlacionadas com a infraestrutura de saúde educação e saúde. Entre os insumos específicos de saúde, apenas a variável Técnico de Enfermagem per capita se mostrou estatisticamente significativa. Porém, esta variável apresentou coeficiente com sinal positivo. Esse resultado não significa que mais técnicos de enfermagem geram mais mortes. Pelo contrário, é possível conjecturar que os municípios onde há maior mortalidade, são aqueles que, em geral, concentram mais os profissionais de menor custo (quando comparados com médicos), desacompanhados dos demais elementos necessários ao bom funcionamento do sistema de saúde. Tal fenômeno decorre da tentativa inócua de atender, de forma precária, a demanda pelo serviço. Então, poder-se-ia especular que os problemas de saúde estejam recaindo sobre profissionais que, sem o apoio e sem a estrutura necessária, são utilizados como substitutos inadequados para os demais insumos necessários para mitigar os complexos problemas de saúde da população brasileira.

Em suma, dado que o objetivo do programa é ofertar serviços de saúde com foco na melhora da saúde da população, via contratação de mais médicos, a conclusão é de que, no contexto desse estudo, não é possível descartar a hipótese de que a motivação do programa está correta. Até porque a presença de médicos deve, em certa medida, reforçar a demanda social, não atendida 
até o momento, pelos demais componentes da infraestrutura necessária ao bom funcionamento de saúde. Isto não significa, porém, que o desenho e a implementação tenham sido feito de modo coerente e condizente com bem-sucedidas experiências similares mundo afora. Muito da polêmica em torno deste programa se dá, justamente, pelo modo que foi feito. As especulações sobre as duas etapas e as chegadas dos médicos cubanos, sem qualquer tipo de programa de inclusão de familiares ${ }^{5}$, ou até mesmo, a precariedade das condições de trabalho às quais os profissionais de saúde podem estar submetidos, suscitam uma série de questionamentos sobre os potenciais resultados deste programa.

Recomenda-se, enfaticamente, que estudos avaliativos do referido programa sejam realizados a partir da observação dos resultados de sua atuação. Para que essas avaliações ocorram, de modo adequado, seria necessário um acompanhamento do mesmo, com a coleta criteriosa e permanente de dados e de informações relevantes. Além disso, seria também imperiosa a publicação de tais resultados, respeitados os limites da ética e do sigilo, para que instâncias e pesquisadores independentes possam, a qualquer tempo, no interesse público, avaliar o Mais Médicos.

5 Dal Poz (2013) destaca que o sucesso das experiências canadenses e australianas se dá, em grande medida, pelo aparato oferecido aos médicos estrangeiros no que se refere à inclusão de familiares - cônjuges e filhos, principalmente - bem como boas condições de trabalho, tanto em termos de remuneração, quanto em termos de infraestrutura. 


\section{Referências bibliográficas}

AFONSO, A.; ST. AUBYN, M. Non-parametric approaches to education and health expenditure efficiency in OECD countries. Journal of applied economics, v. VIII, n. 2, p. 227 246, Nov. 2005

ATLAS DO DESENVOLVIMENTO HUMANO 2013. Programa das Nações Unidas para o Desenvolvimento-PNUD; 2014. Disponível em:

http://www.atlasbrasil.org.br/2013/pt/consulta/

BARROS, R. P. et al. Determinantes do Desenvolvimento na Primeira Infância no Brasil. Texto para Discussão 1478, IPEA Rio de Janeiro, março, 2010.

CAMARANO, A. A.; KANSO, S.; CARVALHO, D.F. Envelhecimento populacional, perda de capacidade laborativa e políticas públicas. Mercado de Trabalbo. Conjuntura e Análise, p. 21-29, 2013.

DATASUS. Cadastro Nacional dos Estabelecimentos de Saúde do Brasil-CNES.

DAL POZ, R. Entrevista à Folha de São Paulo em 04/09/2013.

GONÇALVES, J. A., et al. Ser médico no PSF: formação acadêmica, perspectivas e trabalho cotidiano. Revista Brasileira de Educação Médica, 33 (3), p. 393-403, 2009.

IBGE - CENSOS DEMOGRÁFICOS. DATASUS; 2014 Disponível em: http://www2.datasus.gov.br/DATASUS/ index.php?area $=0206$

JAPAN - WORLD BANK. Japan-Wold Bank Partnership Program on Universal Health Coverage. Global Conference on Universal Health Coverage for Inclusive and Sustainable Growth, Lessons from 11 Country Case Studies: A Global Synthesis, December 5-6, Tokyo, 2013.

LACERDA, A. et al. Reflexão crítica sobre o mercado de trabalho dos médicos no Brasil. Rev. Med. Res., Curitiba, v.14, n.3, p. 193-199, 2012.

MACIEL FILHO, R. Estratégias para a distribuição e fixação de médicos em sistemas nacionais de saúde: o caso brasileiro. Universidade do Estado do Rio de janeiro. Instituto de Medicina Social. Tese de Doutorado em Saúde Coletiva, 2007.

MADDALA, K. Introduction to Econometrics. John Wiley \& Sons Ltd., England, 3rd edition, 2001.
NERI, M.; SOARES, W. Desigualdade social e saúde no Brasil. Cadernos de Saúde Pública, Rio de Janeiro, 18 (Suplemento), p.77-87, 2002

MASSMED - Massachussets Medical Society. Physician Workforce Study, 2010.

MINISTÉRIO DA SAÚDE. Mais Médicos para o Brasil, Mais Saúde para Você, 2013.

MINISTÉRIO DA SAÚDE. Cadastro Nacional dos Estabelecimentos de Saúde do Brasil - CNES. DATASUS; 2014. Disponível em: http://www2.datasus.gov.br/DATASUS/ index.php?area $=0204$

MINISTÉRIO DA SAÚDE Ministério da Saúde. Sistema de Informações sobre Mortalidade - SIM. DATASUS; 2014. Disponível em: http://www2.datasus.gov.br/DATASUS / index.php?area $=0205$

MINISTÉRIO DA SAÚDE. Sistema de Informações sobre Nascidos Vivos - SINASC. DATASUS; 2014. Disponível em: http://www2.datasus.gov.br/DATASUS/index.php?area $=0205$.

MINISTÉRIO DA SAÚDE - Sistema de Informação de Atenção Básica - SIAB. DATASUS; 2014. Disponível em: http:// www2.datasus.gov.br/DATASUS/index.php?ahttp: / /w

PLANALTO FEDERAL. Lei n 12.871 Institui o Programa Mais Médicos, altera as Leis no 8.745, de 9 de dezembro de 1993, e no 6.932, de 7 de julho de 1981, e dá outras providências. Brasília, 2013.

PÓVOA, L.; ANDRADE, M. V. Distribuição geográfica dos médicos no Brasil: uma análise a partir de um modelo de escolha locacional Cadernos de Saúde Pública, Rio de Janeiro, 22(8), p. 1555-1564, 2006.

PREFEITURAS MUNICIPAIS - Sistema de Informacões sobre Orçamentos Públicos em Saúde - SIOPS. DATASUS; 2014. Disponível em:

http://siops-asp.datasus.gov.br/cgi/siops/serhist/MUNICIPIO/indicadores.HTM

OR, Z.; WANG, J; JAMISON, D. International differences in the impact of doctors on health: a multilevel analysis of OECD countries. Journal of Health Economics, v. 24, p. $531-560,2005$ 
Apêndice:

Matriz de correlação

\begin{tabular}{|c|c|c|c|c|c|}
\hline & $\%$ PSF & $\begin{array}{l}\text { Despesa } \\
\text { Per capita }\end{array}$ & $\begin{array}{l}\text { Esperança de } \\
\text { vida ao nascer }\end{array}$ & $\begin{array}{l}\text { Renda per } \\
\text { Capita média }\end{array}$ & IDH \\
\hline$\%$ de cobertura do PSF & 1,000 & & & & \\
\hline Despesa per capita & 0,068 & 1,000 & & & \\
\hline Esperança de vida ao nascer & $-0,177$ & 0,332 & 1,000 & & \\
\hline Renda per capita média & $-0,247$ & 0,336 & 0,784 & 1,000 & \\
\hline IDH & $-0,205$ & 0,375 & 0,852 & 0,908 & 1,000 \\
\hline Expectativa de anos de estudo & $-0,032$ & 0,277 & 0,441 & 0,512 & 0,653 \\
\hline Taxa de analfabetismo - 18 anos ou mais & 0,233 & $-0,336$ & $-0,826$ & $-0,818$ & $-0,886$ \\
\hline Taxa de Mortalidade Infantil & 0,002 & $-0,061$ & $-0,122$ & $-0,125$ & $-0,138$ \\
\hline Equipamentos existentes per capita & $-0,132$ & 0,277 & 0,432 & 0,546 & 0,527 \\
\hline Equipamento SUS per capita & 0,112 & 0,382 & 0,191 & 0,157 & 0,207 \\
\hline Médicos per capita & $-0,190$ & 0,164 & 0,385 & 0,522 & 0,474 \\
\hline Superior enfermeiro per capita & 0,057 & 0,377 & 0,220 & 0,249 & 0,275 \\
\hline Técnicos de enfermagem per capita & $-0,063$ & 0,224 & 0,323 & 0,345 & 0,333 \\
\hline Leitos per capita & $-0,056$ & $-0,042$ & 0,162 & 0,240 & 0,230 \\
\hline \% Água e esgoto adequados & $-0,061$ & 0,316 & 0,604 & 0,587 & 0,668 \\
\hline
\end{tabular}

(Continua)

\begin{tabular}{|c|c|c|c|c|c|}
\hline & $\begin{array}{l}\text { Expectativa } \\
\text { Anos de } \\
\text { Estudo }\end{array}$ & $\begin{array}{l}\text { Taxa } \\
\text { Analfabetismo } \\
18 \text { anos ou mais }\end{array}$ & $\begin{array}{l}\text { Taxa } \\
\text { Mortalidade } \\
\text { Infantil }\end{array}$ & $\begin{array}{l}\text { Equipamentos } \\
\text { Existentes } \\
\text { Per capita }\end{array}$ & $\begin{array}{l}\text { Equipamento } \\
\text { SUS } \\
\text { Per capita }\end{array}$ \\
\hline $\begin{array}{l}\text { Expectativa de anos de } \\
\text { estudo }\end{array}$ & 1,000 & & & & \\
\hline $\begin{array}{l}\text { Taxa de analfabetismo - } \\
18 \text { anos ou mais }\end{array}$ & $-0,492$ & 1,000 & & & \\
\hline $\begin{array}{l}\text { Taxa de Mortalidade } \\
\text { Infantil }\end{array}$ & $-0,113$ & 0,117 & 1,000 & & \\
\hline $\begin{array}{l}\text { Equipamentos existen- } \\
\text { tes per capita }\end{array}$ & 0,273 & $-0,445$ & $-0,060$ & 1,000 & \\
\hline $\begin{array}{l}\text { Equipamento SUS per } \\
\text { capita }\end{array}$ & 0,166 & $-0,173$ & $-0,014$ & 0,618 & 1,000 \\
\hline Médicos per capita & 0,179 & $-0,390$ & $-0,052$ & 0,542 & 0,118 \\
\hline $\begin{array}{l}\text { Superior enfermeiro per } \\
\text { capita }\end{array}$ & 0,205 & $-0,202$ & $-0,022$ & 0,381 & 0,316 \\
\hline $\begin{array}{l}\text { Técnicos de Enferma- } \\
\text { gem per capita }\end{array}$ & 0,133 & $-0,338$ & $-0,009$ & 0,382 & 0,233 \\
\hline Leitos per capita & 0,137 & $-0,174$ & $-0,027$ & 0,342 & 0,173 \\
\hline $\begin{array}{l}\text { \% Água esgoto ade- } \\
\text { quados }\end{array}$ & 0,443 & $-0,585$ & $-0,082$ & 0,343 & 0,205 \\
\hline
\end{tabular}

(Continua) 
Matriz de correlação

\begin{tabular}{|c|c|c|c|c|c|}
\hline & $\begin{array}{l}\text { Médicos } \\
\text { Per capita }\end{array}$ & $\begin{array}{l}\text { Superior } \\
\text { Enfermeiro } \\
\text { Per capita }\end{array}$ & $\begin{array}{l}\text { Técnico } \\
\text { Enfermagem } \\
\text { Per capita }\end{array}$ & $\begin{array}{l}\text { Leitos } \\
\text { Per capita }\end{array}$ & $\begin{array}{l}\text { \% Água esgoto } \\
\text { Adequado }\end{array}$ \\
\hline $\begin{array}{l}\text { Médicos per } \\
\text { capita }\end{array}$ & 1,000 & & & & \\
\hline $\begin{array}{l}\text { Superior enfer- } \\
\text { meiro per capita }\end{array}$ & 0,369 & 1,000 & & & \\
\hline $\begin{array}{l}\text { Técnicos de } \\
\text { enfermagem per } \\
\text { capita }\end{array}$ & 0,383 & 0,338 & 1,000 & & \\
\hline Leitos per capita & 0,352 & 0,263 & 0,281 & 1,000 & \\
\hline $\begin{array}{l}\text { \% Água e esgoto } \\
\text { adequados }\end{array}$ & 0,274 & 0,213 & 0,200 & 0,134 & 1,000 \\
\hline
\end{tabular}

Descrição das variáveis

\begin{tabular}{|c|c|c|c|c|c|c|c|}
\hline Variáveis & Obs. & Média & Desvio Padrão & Min. & Max & \multicolumn{2}{|c|}{ Intervalo de confiança $95 \%$} \\
\hline$\%$ de cobertura do PSF & 5565 & 83,415 & 43,64 & 0 & 393,52 & 82,268 & 84,562 \\
\hline Despesa per capita & 5565 & 383,945 & 174,41 & 0 & 1980,19 & 379,362 & 388,529 \\
\hline $\begin{array}{l}\text { Esperança de vida ao } \\
\text { nascer }\end{array}$ & 5565 & 73,089 & 2,681 & 65,3 & 78,64 & 73,019 & 73,159 \\
\hline Renda per capita média & 5565 & 493,606 & 243,269 & 96,25 & 2043,74 & 487,213 & 499,999 \\
\hline IDH & 5565 & 0,659 & 0,072 & 0,418 & 0,862 & 0,657 & 0,661 \\
\hline $\begin{array}{l}\text { Expectativa de anos de } \\
\text { estudo }\end{array}$ & 5565 & 9,464 & 1,098 & 4,34 & 12,83 & 9,435 & 9,493 \\
\hline $\begin{array}{l}\text { Taxa de analfabetismo - } \\
18 \text { anos ou mais }\end{array}$ & 5565 & 17,405 & 10,699 & 0,97 & 47,64 & 17,123 & 17,686 \\
\hline $\begin{array}{l}\text { Taxa de Mortalidade } \\
\text { Infantil }\end{array}$ & 5565 & 14,26 & 14,282 & 0 & 333,333 & 13,885 & 14,636 \\
\hline $\begin{array}{l}\text { Equipamentos existentes } \\
\text { per capita }\end{array}$ & 5565 & 2,976 & 2,623 & 0 & 41,143 & 2,907 & 3,045 \\
\hline $\begin{array}{l}\text { Equipamento SUS } \\
\text { per capita }\end{array}$ & 5565 & 1,579 & 1,227 & 0 & 12,5 & 1,546 & 1,611 \\
\hline Médicos per capita & 5565 & 0,643 & 0,598 & 0 & 5,945 & 0,628 & 0,659 \\
\hline $\begin{array}{l}\text { Superior enfermeiro } \\
\text { per capita }\end{array}$ & 5565 & 0,551 & 0,262 & 0 & 3,632 & 0,544 & 0,558 \\
\hline $\begin{array}{l}\text { Técnicos de Enfermagem } \\
\text { per capita. }\end{array}$ & 5565 & 0,726 & 0,744 & 0 & 6,513 & 0,706 & 0,745 \\
\hline Total de Leitos per capita & 5565 & 1,854 & 2,178 & 0 & 31,289 & 1,797 & 1,912 \\
\hline $\begin{array}{l}\text { \% Água e esgoto } \\
\text { adequados }\end{array}$ & 5565 & 90,798 & 12,839 & 14,64 & 100 & 90,461 & 91,136 \\
\hline
\end{tabular}




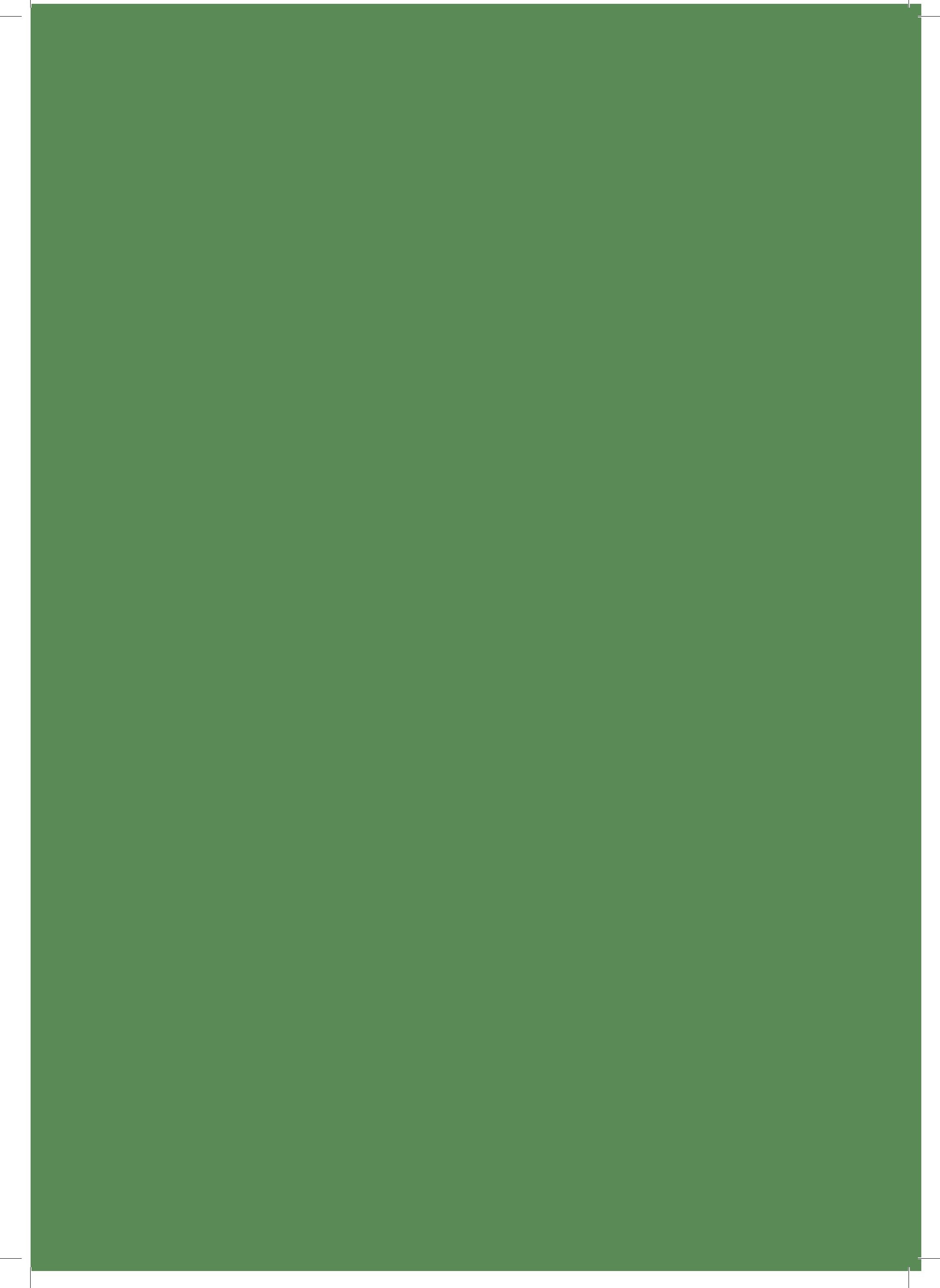

